\title{
APLICAÇÕES TERAPÊUTICAS DA ESPÉCIE Dipteryx alata Vogel
}

Claudiane Marques Ferreira $^{1}$, Nayane Peixoto Soares ${ }^{2}$, Vanessa de Souza Vieira ${ }^{3}$, Vanessa de Sousa Cruz ${ }^{4}$,Eugênio Gonçalves de Araújo ${ }^{5}$

1 Mestranda Programa de Pós-Graduação em Ciência Animal da Escola de Veterinária e Zootecnia da Universidade Federal de Goiás (marquesveterinaria@gmail.com)

2 Doutoranda do Programa de Pós-Graduação em Ciência Animal da Escola de Veterinária e Zootecnia da Universidade Federal de Goiás

3 Doutoranda do Programa de Pós-Graduação em Ciência Animal da Escola de Veterinária e Zootecnia da Universidade Federal de Goiás

4 Pós-doutoranda, Laboratório Multiusuário de Avaliação de Moléculas, Células e Tecidos da Universidade Federal de Goiás-Brasil

5 Professor Doutor do Setor de Patologia Veterinária da Universidade Federal de Goiás

\section{Recebido em: 22/09/2018 - Aprovado em: 23/11/2018 - Publicado em: 03/12/2018} DOI: 10.18677/EnciBio_2018B8

\begin{abstract}
RESUMO
Objetivou-se com essa revisão de literatura avaliar as implicações terapêuticas de $D$. alata relatadas na literatura. A amêndoa de $D$. alata foi utilizada em pesquisas com ratos e camundongos para avaliações de perfil lipídico e como antioxidante. Os resultados são promissores e comprovaram que o consumo diário da semente, mesmo em pequenas doses melhora algumas análises clínicas como glicemia e colesterol. A divulgação destes resultados pode contribuir para um aumento do consumo da semente, proporcionando aumento na produção e comercialização da amêndoa podendo proporcionar um complemento na renda de produtores rurais e ainda uma melhora significativa na saúde humana. Pode haver uma contribuição na redução de doenças cardiovasculares. Estudos com as amêndoas em humanos também comprovaram redução em LDL-c devido a presença de ácidos graxos insaturados e compostos bioativos. De todas as implicações terapêuticas pesquisadas com $D$. alata, nenhuma demonstrou a citotoxicidade de partes da planta em estudos cancerígenos e nem todas as partes da espécie foram utilizadas em pesquisas, como o endocarpo do fruto. Assim, tornam-se necessário e primordial estudos de outras partes do fruto de $D$. alata, bem como novos estudos referentes às implicações terapêuticas dessa importante espécie na área rural de Goiás.
\end{abstract}

PALAVRAS-CHAVE: baru, fenois, antioxidante.

\section{THERAPEUTIC APPLICATIONS OF THE SPECIES Dipteryx alata Vogel}

\begin{abstract}
The aim of this literature review was to evaluate the therapeutic implications of $D$. alata reported in the literature. The almond of $D$. alata was used in research with rats and mice for evaluations of lipid profile and as antioxidant. The results are promising and have shown that the daily consumption of the seed, even in small doses improves some clinical analyzes such as glycemia and cholesterol. The dissemination of these results can contribute to an increase in the consumption of the
\end{abstract}


seed, providing an increase in the production and commercialization of the almond, being able to provide a complement in the income of rural producers and still a significant improvement in the human health. There may be a contribution to reducing cardiovascular disease. Studies with almonds in humans have also shown a reduction in LDL-C due to the presence of unsaturated fatty acids and bioactive compounds. Of all the therapeutic implications investigated with $D$. alata, none have demonstrated the cytotoxicity of parts of the plant in carcinogenic studies and not all parts of the species are used in research, such as the endocarp of the fruit. Thus, it is necessary and primordial studies of other parts of the fruit of $D$. alata, as well as new studies regarding the therapeutic implications of this important species in the rural area of Goiás.

KEYWORDS: baru, fenois, antioxidant.

\section{INTRODUÇÃO}

As pesquisas com espécies do Cerrado são consideradas um importante meio de divulgação desses recursos biológicos objetivando a conservação, e ainda podem contribuir para a disseminação do conhecimento sobre seus benefícios para a saúde humana. Em termos de biodiversidade foi identificado como um dos hotspots de biodiversidade mundial, tendo cerca de 4400 espécies de plantas endêmicas (FANK-de-CARVALHO et al., 2015). Contudo, são poucos os investimentos em conservação e pesquisa, o que proporciona lacunas na caracterização da flora desse bioma. Algumas espécies do Cerrado possuem potencial para servirem como fontes de substâncias bioativas, objetivando potenciais medicinais como os compostos fenólicos. Estes já foram identificados em Dipteryx alata Vogel justificando estudos terapêuticos em doenças crônicas.

A D. alata é utilizada, na cultura popular, como antirreumático, regulador menstrual e redutor de taxas de colesterol (SOUZA; FELFILI, 2006; ESTEVESPEDRO et al., 2012). A utilização de plantas em tratamentos é milenar e ocorre em regiões pobres do país, mas podem ser comercializadas em feiras e mercados populares de grandes cidades. Algumas populações não possuem acesso a drogas sintéticas, portanto a terapia herbal é utilizada de forma cultural (SILVA et al., 2011). Apesar de não terem seus compostos bioativos conhecidos, as observações populares dos efeitos contribuem para as pesquisas sobre aplicações terapêuticas no mundo todo (MACIEL et al., 2002).

A partir dos anos 80 e 90 houve um resgate do uso terapêutico das plantas medicinais no meio científico, com o objetivo de atuação como meio de complementar as práticas médicas utilizadas e não como contraposição de práticas alopáticas. Alguns fatos motivaram este resgate como, por exemplo, a falta de êxito no modelo médico biologicista no tratamento de doenças e a comprovação científica de algumas plantas no tratamento de doenças. Existe uma tentativa de legitimação da utilização do uso terapêutico de plantas medicinais que antes estava à margem das instituições de saúde (ALVIM et al., 2006).

Como parte da atenção primária à saúde, cerca de $80 \%$ da população mundial utiliza produtos fitoterápicos, muitos com o objetivo de promover uma vida mais saudável (EKOR, 2014). O consumo de fitoterápicos valida conhecimento popular repassados por gerações e isto desperta interesse em pesquisadores envolvidos em áreas do conhecimento sobre a fonte medicinal natural (MACIEL et al., 2002). As espécies do Cerrado brasileiro são utilizadas pela comunidade local como fonte de alimento e como remédio (BAILÃO et al., 2015). Diante disso, 
objetivou-se com essa revisão de literatura avaliar as implicações terapêuticas de $D$. alata relatadas na literatura.

\section{APLICAÇÃO TERAPÊUTICA DA AMÊNDOA}

Entre as principais causas de mortes em humanos estão as doenças crônico-degenerativa não transmissíveis e dentre estas estão inclusas as complicações cardiovasculares e a síndrome metabólica. O consumo de amêndoas é considerado benéfico na prevenção destas condições, especialmente devido a composição em ácidos graxos insaturados e outros compostos bioativos como os fenois. Devido ao sabor agradável e do alto valor nutricional a amêndoa de D. alata tem tido seu consumo aumentado (ARAÚJO et al., 2016; WU et al., 2017; WONG et al., 2016) e alguns pesquisadores iniciaram sua utilização para avaliar os efeitos benéficos.

Em um estudo com camundongos Swiss machos induzidos a obesidade hiperglicídica durante 60 dias a amêndoa do $D$. alata foi utilizada na dieta e os níveis séricos de glicose e triglicérides foram reduzidos. Ao final do experimento, os animais foram pesados e houve um menor ganho de peso no grupo tratado com a $D$. alata, mesmo não havendo diferença na ingesta de dietas entre os grupos, comprovando que o consumo da dieta com amêndoa de $D$. alata não diminuiu o apetite dos animais. Não houve diferença significativa na redução dos níveis séricos de colesterol e na quantidade de tecido adiposo nos animais que receberam $D$. alata (Figura 1). Estudos como esse contribuem para a divulgação do consumo de amêndoas em comunidades remotas do cerrado e proporcionam melhoria na dieta dessa população (ARAÚJO et al., 2017).

Em uma pesquisa com ratos Wistar os pesquisadores avaliaram o perfil metabólico e oxidativo após a administração de ração com amêndoas de $D$. alata em grupos divididos por porcentagem de ração, sendo um grupo sem a suplementação, outros com $20 \%, 30 \%$ e $40 \%$ de suplementação. O consumo foi ad libitum para todos os grupos além de fornecimento de água. A eutanásia dos animais ocorreu após 40 dias de tratamento e foram coletadas amostras de sangue e avaliados perfil lipídico e índices aterogênicos. Houve redução considerável nos índices de glicemia, colesterol, triglicerídeos, lipoproteína de baixa densidade-colesterol. Não houve redução nos níveis de colesterol. Por não haver aumento nos níveis de AST (aspartato aminotransferase) e ALT (alanina aminotransferase) os autores acreditam que não houve danos em células do fígado indicando que a amêndoa é segura para consumo. Em resumo a utilização de amêndoa na alimentação pode auxiliar na prevenção de doenças cardiovasculares porque houve redução significativa de razão de risco cardíaco, no coeficiente e índice aterogênico (FIORINI et al., 2017).

Em uma comparação de consumo de amêndoas de baru, castanha do Brasil e dieta hiperlipídicas com $7 \%$ de óleo de soja em ratos Wistar foram avaliados para os níveis séricos de colesterol total (CT), triacilglicerol (TG) e colesterol de lipoproteína de alta densidade (HDL-c). Além de análise de malonodialdeído (MDA) e vitamina $\mathrm{E}$ hepática. As castanhas preveniram peroxidação lipídica no tecido hepático e hiperlipidemia, o que os autores correlacionaram com um papel protetor de ambas contra a alta ingestão de colesterol devido aos altos níveis de ácidos graxos insaturados (baru $70 \mathrm{~g} / 100 \mathrm{~g}$, castanha do Brasil $77 \mathrm{~g} / 100 \mathrm{~g}$ ). Entre as amêndoas o baru demonstrou melhores resultados em CT (119,33 baru, 126,50 castanha do Brasil), triacilglicerol (57 baru e 60,67 castanha do Brasil), HDL-c (23 
baru e 36,33 castanha do Brasil). MDA teve melhores resultados com a castanha do Brasil $(92,31)$ comparada ao baru $(153,65)$ (FERNANDES et al., 2015).

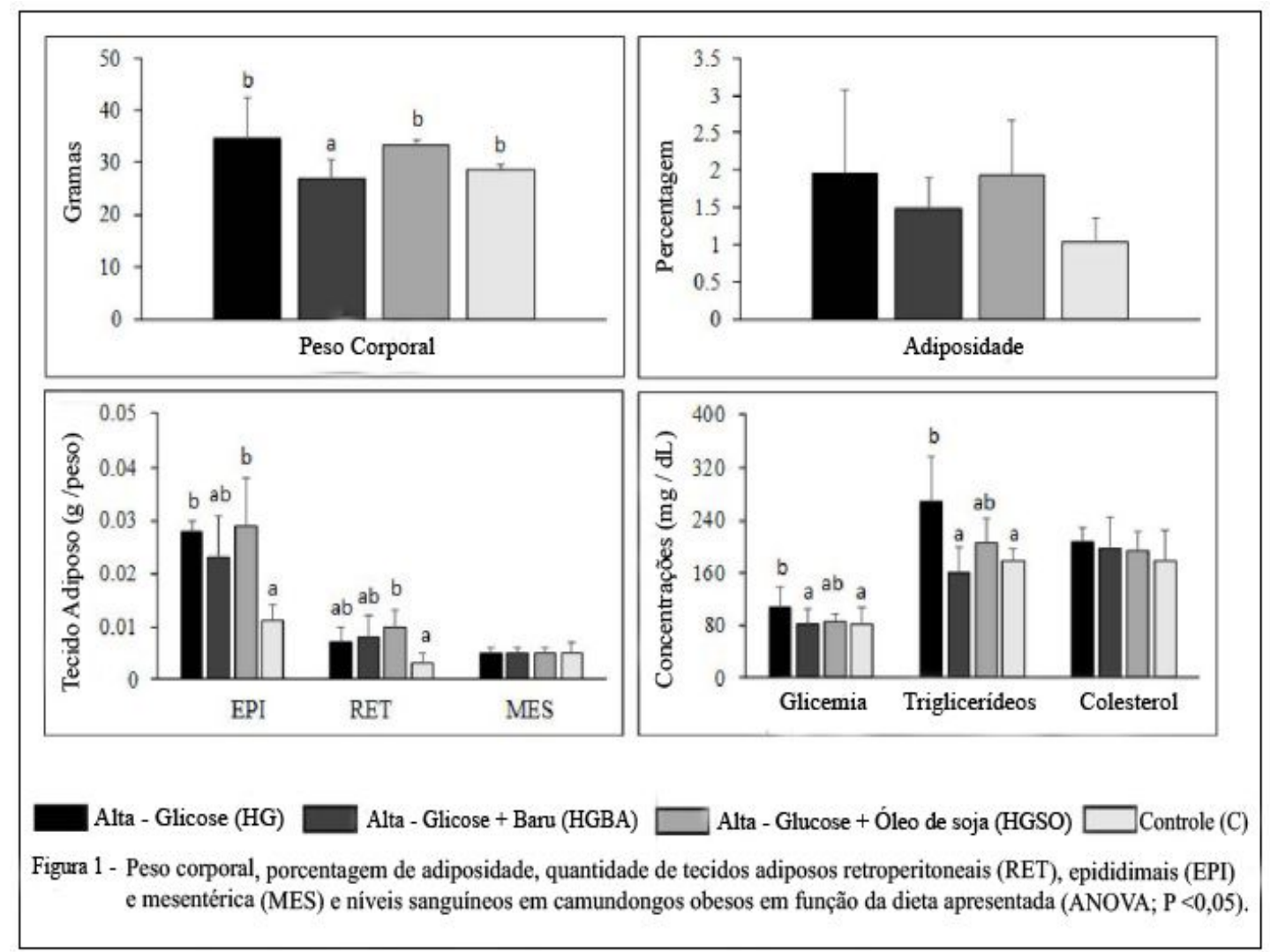

FIGURA 1- Resultados de camundongos Seiss com sete indivíduos em cada grupo sendo um grupo com ração de altos níveis de energia $(\mathrm{HG})$, com altos níveis de glicose acrescido de amêndoa de baru (HGBA), dieta com altos níveis de glicose acrescido de óleo de soja (HGSO) e um grupo controle alimentados com ração para camundongos Labina®. No quadro A - observa-se um menor ganho de peso em animais alimentados com ração de níveis altos de glicose acrescido com a amêndoa do baru. No quadro B - não observou menores níveis de acúmulo de tecido adiposo em retroperitoneal (RET), epididimal (EPI) e mesentérica (MES) com a ração HGBA em comparação com o grupo controle. No quadro C - observa-se redução nos níveis séricos de glicose e triglicerídeos, mas não no nível de colesterol comparado ao grupo controle.

Fonte: Adaptado de Araújo et al., (2017).

A vitamina $E$ hepática, principalmente na forma de $\alpha$-tocoferol, atuando como um antioxidante eficaz, é capaz de bloquear o início ou inibindo a propagação de Peroxidação de lipídios. Os maiores níveis de vitamina $E$ hepática estavam nos fígados de animais suplementados com ambas amêndoas (FERNANDES et al., 2015).

Também utilizando as amêndoas de $D$. alata, um grupo de pesquisadores suplementou ratos com doses de Ferro (Fe) para induzir o estresse oxidativo e investigar a ação da semente como antioxidante. O Fe constitui papel importante no metabolismo celular participando da síntese de DNA e respiração celular, mas pode na forma livre realizar catálise das reações de oxidação de moléculas podendo ENCICLOPÉDIA BIOSFERA, Centro Científico Conhecer - Goiânia, v.15 n.28; p.84 
causar toxicidade aguda nos rins e fígado. Foram utilizadas amêndoas torradas sem casca (100 g kg-1) como suplemento na ração associado ou não a Fe em ratos Wistar. Após 17 dias, a suplementação oral de Fe resultou em aumento da oxidação de lipídios no fígado e no baço e a amêndoa pôde ser indicada pela indução da redução do dano. O mesmo parece acontecer em tecidos com danos proteicos reduzindo os radicais livres e não quelando o $\mathrm{Fe}$ e os autores atribuem essas ações antioxidativas à presença de compostos fenólicos principalmente taninos (SIQUEIRA et al., 2012).

Uma análise de $D$. alata em humanos analisou o efeito do acréscimo de amêndoa $(20 \mathrm{~g})$ em dietas de indivíduos com hipercolesterolemia leve e demonstrou redução do colesterol total $(C T)$ e a lipoproteína de baixa densidade (LDL-c) séricos, fatores que podem contribuir com a diminuição do risco de doenças cardiovasculares tendo em vista que o consumo de amêndoas pode melhorar o perfil lipídico sérico e reduzir o estresse oxidativo. Vinte pessoas, com idades entre $21 \mathrm{e}$ 57 anos, foram avaliadas por meio da administração de $20 \mathrm{~g}$ de amêndoa de $D$. alata torradas e cápsula de amido de milho (como placebo). A designação do tratamento foi aleatória e os indivíduos receberam um dos dois tratamentos em períodos alternados por seis semanas cada um, com um intervalo de quatro semanas entre os tratamentos (BENTO et al., 2014).

Todos os sujeitos do estudo receberam a informação que a dieta suplementar seria com amêndoas de $D$. alata processadas de duas maneiras diferentes: amêndoas torradas e amêndoas em cápsula. O período de seis semanas foi escolhido por ser o tempo considerado suficiente para estabilização de lipoproteínas induzidas na dieta. O intervalo de quatro semanas foi proposto como transição com o objetivo de eliminar os efeitos residuais do primeiro período. As amêndoas foram adquiridas em comércio local em Goiânia, oriundas de Pirenópolis. Estas foram torradas com intuito de investigar o efeito de seu consumo habitual. Após seis semanas de consumo, mesmo com aumento de $110 \mathrm{kcal} / \mathrm{dia}$ em suas refeições diárias pelo consumo da amêndoa os indivíduos não obtiveram aumento de peso corporal. Os autores concluíram que mesmo com o incremento na quantidade de calorias diárias a alta quantidade de fibras e proteínas na amêndoa da $D$. alata, promoveu a diminuição de ingestão de outros alimentos mantendo a ingestão usual (BENTO et al., 2014).

Por meio de duas análises sanguíneas, ambas realizadas com os indivíduos em jejum de 12 horas, no início do estudo e no final não demonstraram redução em HDL-c (lipoproteína de alta densidade), relação CT/HDL-c e LDL-c/HDLc, porém houve redução nos níveis de CT e LDL-c quando comparado ao período de ingestão do placebo (BENTO et al., 2014). (Tabela 1).

TABELA 1 - Composição corporal dos indivíduos e lipídios séricos no dia inicial (base) e após seis semanas de tratamento com amêndoas de $D$. alata e placebo $(n=20)$.

\begin{tabular}{lllll}
\hline PARÂMETROS & AMÊNDOA & & PLACEBO & \\
& BASE & 6 SEMANAS & BASE & 6 SEMANAS \\
\hline Peso, $\mathrm{kg}$ & $64,0 \pm 2,4$ & $63,8 \pm 2,5$ & $63,4 \pm 2,6$ & $63,8 \pm 2,6$ \\
$\mathrm{HDL}-\mathrm{c}, \mathrm{mmol} / \mathrm{L}$ & $1,4 \pm 0,1$ & $1,3 \pm 0,1$ & $1,3 \pm 0,1$ & $1,3 \pm 0,1$ \\
$\mathrm{LDL}-\mathrm{c}, \mathrm{mmol} / \mathrm{L}$ & $4,0 \pm 0,2$ & $3,7 \pm 0,2$ & $3,7 \pm 0,2$ & $3,8 \pm 7,8$ \\
$\mathrm{CT}, \mathrm{mmol} / \mathrm{L}$ & $6,0 \pm 0,2$ & $0 \pm 0,1$ & $5,6 \pm 0,2$ & $5,7 \pm 0,2$ \\
\hline
\end{tabular}


Para diminuição dos riscos de desenvolvimento de doenças cardiovasculares, a concentração de LDL-c torna-se mais importante por estar diretamente envolvida na gênese da aterosclerose uma das principais causas de doenças cardiovasculares. Os autores acreditam que a redução de LDL-c deve-se ao fato da presença de ácidos graxos insaturados, fibras e compostos bioativos na amêndoa da $D$. alata. O ácido oleico presente na amêndoa pode reduzir LDL-c por diminuir a susceptibilidade destas partículas à oxidação. As fibras podem limitar a absorção intestinal do colesterol da alimentação por formar géis (BENTO et al., 2014).

Outro estudo em humanos avaliou dieta rica em amêndoa de baru para avaliar a redução de adiposidade abdominal e concentrações de lipoproteínas. Em um ensaio clínico randomizado, controlado por placebo, com 86 mulheres, entre 20 e 59 anos, com sobrepeso e obesas, foram introduzidas duas dietas na alimentação das participantes com instruções dietéticas, uma dieta com amêndoa de baru, e uma sem a amêndoa. Ambas as dietas continham entre 25 a $30 \mathrm{kcal} / \mathrm{kg}$. Os autores justificaram a utilização da amêndoa pela associação da diminuição dos riscos de doenças cardiovasculares, e a amêndoa do baru foi escolhida para o estudo por conter alto teor de proteínas e alta qualidade de ácidos graxos mono e poliinsaturados, além de fenois (SOUZA et al., 2018).

Os resultados foram melhores com a introdução da amêndoa de baru na dieta e as avaliações consistiram em marcadores de metabolismo lipídico e composição corporal. Antes e após oito semanas de consumo das duas dietas as participantes foram submetidas a exames de aferição da pressão arterial, perfil lipídico, ácidos graxos plasmáticos e níveis de glicose. Além de avaliações da expressão de proteína de transferência de éster de colesterila, angiopoietina-like-3 e apolipoproteínas. As avaliações corporais consistiram em absortometria radiológica de dupla energia e antropometria (SOUZA et al., 2018).

O grupo que ingeriu as amêndoas de baru teve redução de lipoproteína de alta densidade $(-5,63 \%)$ e de baixa densidade $(-7,62 \%)$, glicemia $(-6,06 \%)$, triacilglicerídeos $(-11,47 \%)$ e em circunferência da cintura em 1,73 vezes maior do que o grupo sem ingestão de baru. Não houve alterações em níveis de apolipoproteínas, expressão de proteína de transferência de éster de colesterila, angiopoietina-like-3 e em aferições de pressão arterial. Os autores concluíram que o estudo trouxe dados positivos inferindo que o consumo de amêndoas de baru, durante oito semanas, mesmo em pequenas quantidades conseguiu reduzir marcadores de metabolismo lipídico em mulheres e melhorou a lipoproteína de alta densidade e ainda a adiposidade abdominal (SOUZA et al., 2018).

Com o óleo da amêndoa de D. alata um outro grupo de pesquisadores desenvolveu uma emulsão em fase de gel lamelar com a finalidade de desenvolver veículos para drogas e agentes cosméticos de pele. O óleo foi escolhido por possuir altos terrores de fitoesteróis que auxiliam no potencial econômico do uso desta espécie como matéria prima na indústria farmacêutica e cosmética. Vários testes foram utilizados e os autores chegaram à conclusão que o sistema desenvolvido pode ser bom para os tratamentos de pele, especialmente porque o óleo foi capaz de extrair um marcador de spin de ácido graxo das estruturas de domínio lipídico de subcutâneo da pele (MORAES et al., 2018). 


\section{APLICAÇÃO TERAPÊUTICA DA FOLHA}

O uso de compostos fenólicos como antioxidantes também tem sido utilizado em doenças como hiperpigmentação da pele, que pode ser dependente do aumento da atividade melanogênica de enzimas como a tirosinase, por ser considerada enzima chave na coloração da pele. Compostos inibidores e ativadores da tirosina têm sido utilizados, o primeiro para clarear a pele e o segundo com intuito de aumentar a melanogênese. Baseado em outros estudos com extratos de plantas, uma pesquisa avaliou o extrato da folha da $D$. alata para analisar a capacidade de inibição da tirosinase (SILVERIO et al., 2013).

O ensaio com o extrato etanólico demonstrou $42 \%$ de atividade inibitória após uma hora, e o controle positivo com ácido Kójico inibiu completamente a tirosinase. Acredita-se que os compostos fenólicos competem com a tirosina, substrato da tirosinase, por terem estruturas semelhantes. Apesar de não apresentar uma redução de $100 \%$, os autores acreditam que novos estudos devem ser direcionados para o extrato etanólico da folha do $D$. alata com o objetivo de isolar, quantificar as substâncias presentes e realizar ensaios biológicos mais complexos, como a utilização em culturas de melanócitos (SILVERIO et al., 2013).

\section{APLICAÇÃO TERAPÊUTICA DE CASCA, POLPA E AMÊNDOA}

Um estudo de extratos hidroalcoólicos com a casca, polpa e semente de D. alata, Genipa americana L. (jenipapo) e Vitex cymosa Bert. (tarumã), avaliou as atividades antimicrobianas e antifúngica. Os extratos permaneceram incubados com Staphylococcus aureus, Escherichia coli e Pseudomonas aeruginosa a $35^{\circ} \mathrm{C}$, durante 24h. Com Cândida albicans a incubação foi de $42 \mathrm{~h}$ em $27^{\circ} \mathrm{C}$ (SANTOS et al., 2017). O extrato hidroalcoólico da polpa do $D$. alata mostrou maiores inibições contra $S$. aureus, mas sem diferença quando comparado ao antibiótico Vancomicina. Contra E. coli, o extrato da casca demonstrou melhor resultado e contra $C$. albicans o extrato da semente atingiu níveis melhores (Tabela 2). Os compostos fenólicos foram implicados na ação de $D$. alata contra as bactérias e fungos, por já terem sido identificados na semente (PUEBLA et al., 2010). De todos os frutos, os extratos das partes do jenipapo demonstraram melhores resultados (SANTOS et al., 2017).

TABELA 2 - Estudo com extrato hidroalcoólico de casca, polpa e semente de Dipteryx alata Vogel contra S. aureus, E. coli, P. aeruginosa e C. albicans em teste de difusão de disco

\begin{tabular}{lllll}
\hline $\begin{array}{l}\text { Extrato } \\
\text { alata }\end{array}$ & $D$. & \multicolumn{4}{l}{ Microorganismos } \\
S. aureus & E. coli & P. aeruginosa & C. albicans \\
\hline $10 \%$ & $3.33 \pm 0,33$ & $0.17 \pm 0.17$ & $0.17 \pm 0.17$ & $0.17 \pm 0.17$ \\
$20 \%$ & $3.17 \pm 0,31$ & $1.50 \pm 0.22$ & $0.67 \pm 0.21$ & $0.17 \pm 0.17$ \\
$30 \%$ & $2.67 \pm 0.21$ & $3.00 \pm 0.37$ & $1.17 \pm 0.17$ & $0.17 \pm 0.17$ \\
$10 \%$ & $4.00 \pm 0.45$ & $0.17 \pm 0.17$ & $1.17 \pm 0.17$ & $0.17 \pm 0.17$ \\
$20 \%$ & $4.00 \pm 0.26$ & $0.17 \pm 0.17$ & $1.17 \pm 0.17$ & $0.67 \pm 0.21$ \\
$30 \%$ & $4.00 \pm 0.22$ & $0.17 \pm 0.17$ & $1.17 \pm 0.17$ & $1.17 \pm 0.17$ \\
$10 \%$ & $0.33 \pm 0.21$ & $0.17 \pm 0.17$ & $0.17 \pm 0.17$ & $0.67 \pm 0.21$ \\
$20 \%$ & $0.50 \pm 0.22$ & $0.17 \pm 0.17$ & $0.17 \pm 0.17$ & $1.17 \pm 0.17$ \\
$30 \%$ & $0.67 \pm 0.21$ & $0.17 \pm 0.17$ & $0.17 \pm 0.17$ & $1.67 \pm 0.21$ \\
\hline
\end{tabular}

Fonte: Santos et al., (2017). 


\section{APLICAÇÃO TERAPÊUTICA DA CASCA DO CAULE}

Com o extrato hidroalcoólico da casca da árvore da $D$. alata, com $3,66 \%$ de polifenóis, foram realizados testes de mutagenicidade in vitro, pelo teste de Ames, com cepas de Salmonella typhimurium (TA100, TA98, TA97, TA102) com o objetivo de testar a toxicidade do extrato. As concentrações do extrato de $D$. alata foram de $3.6,7.1,14.1$ e $21.2 \mathrm{mg} /$ placa e o extrato não foi mutagênico para nenhuma das cepas avaliadas (ESTEVES-PEDRO et al., 2012).

No mesmo estudo, foi avaliada a segurança do extrato hidroalcoólico da casca in vivo, justificado pelo consumo humano do chá da casca, principalmente durante a gestação. Foram utilizados ratos Wistar masculinos e femininos, o acasalamento ocorreu e a gravidez foi confirmada. As fêmeas ficaram alojadas em grupos de sete animais, recebendo extrato hidroalcoólico por gavage. Um grupo recebeu $0,5 \mathrm{~g} / \mathrm{kg}$ dia extrato da casca e o outro água deionizada dos dias 0 a $22 \mathrm{da}$ gestação. Os parâmetros avaliados foram a avaliação da capacidade reprodutiva, ganho de peso de ratas gestantes, efeitos de sobrevivência na prole e desenvolvimento neurocomportamental dos filhotes. $O$ resultado foi um perfil de segurança no consumo do extrato hidroalcoólico da casca do caule nos ratos e em seus filhotes (ESTEVES-PEDRO et al., 2012).

Da casca do caule de $D$. alata foram extraídos sub-extratos de hexano (Hex), diclometano (Dcm), acetato de etilo (Eac) e metanol (Met), contendo triterpenoides, isoflavonoides, chalconas e três compostos fenólicos: ácido vanilico, vanilina e ácido procatequina, todos confirmados por cromatografia em camada. Os subextratos foram testados contra os efeitos de bloqueio neuromuscular de veneno de Bothrops jararacussu (Bjssu). Os ensaios farmacológicos foram realizados em preparações de nervo frênico-diafragma de rato determinando a resposta de contração sob estímulos elétricos indiretos após a incubação. Foram utilizados 40 $\mu \mathrm{g} / \mathrm{mL}$ de Bjssu e $50 \mu \mathrm{g} / \mathrm{mL}$ de cada subextratos (PUEBLA et al., 2010).

Os resultados demonstraram que os extratos de Met e Dcm impediram $100 \%$ do bloqueio neuromuscular do veneno de Bjssu. Após 20 minutos, houve diferença na ação de Dcm, Eac e Met. De todos os subextratos, o Dcm demonstrou proteção mais eficaz (Figura 2) e seus resultados em cromatografia em camada quantificaram os compostos fenólicos ácido vanílico, 3',78-tri-hidroxi-4'-metoxiisoflavona e $3^{\prime}, 4^{\prime}, 6$-tri-hidroxi-inurona que foram implicados como substâncias que agiram contra o veneno. Contudo, não houve esclarecimento sobre o mecanismo de ação desses compostos e outros estudos demonstraram que os compostos bioativos apresentam melhor eficácia em sinergia ou em antagonia com outros compostos (PUEBLA et al., 2010).

Em outro estudo foram testados extratos com o veneno de Bjuss e de mais uma espécie, Crotalus durissus terrificus (Cd). Os autores justificaram a utilização em venenos de duas espécies por cada um poder apresentar substâncias, mecanismo de ação e locais de atuações diferentes. Da casca do caule foram produzidos subextratos Met, Dcm, Hex e Eac e a partir dos quatro produziram o extrato hidroalcoólico (HA) com etanol a 70\%. O HA foi submetido a precipitação de proteínas para separar os taninos, já comprovados como inibidor de veneno de outras espécies gerando dois novos extratos: $\mathrm{HA}+$ precipitado de proteínas com presença de tanino e o HA- sobrenadante sem a presença de taninos. Os dois subextratos foram incubados em veneno Bjssu e inoculados em nervo frênicodiafragma de rato e estimulados indiretamente. Os dois extratos melhoraram a tensão da contração muscular, resultando na diminuição do bloqueio neuromuscular 
causado pelo veneno de Bjuss. Comparando a ação dos dois extratos, HA+ demonstrou melhor efeito (NAZATO et al., 2010) (Figura 3).

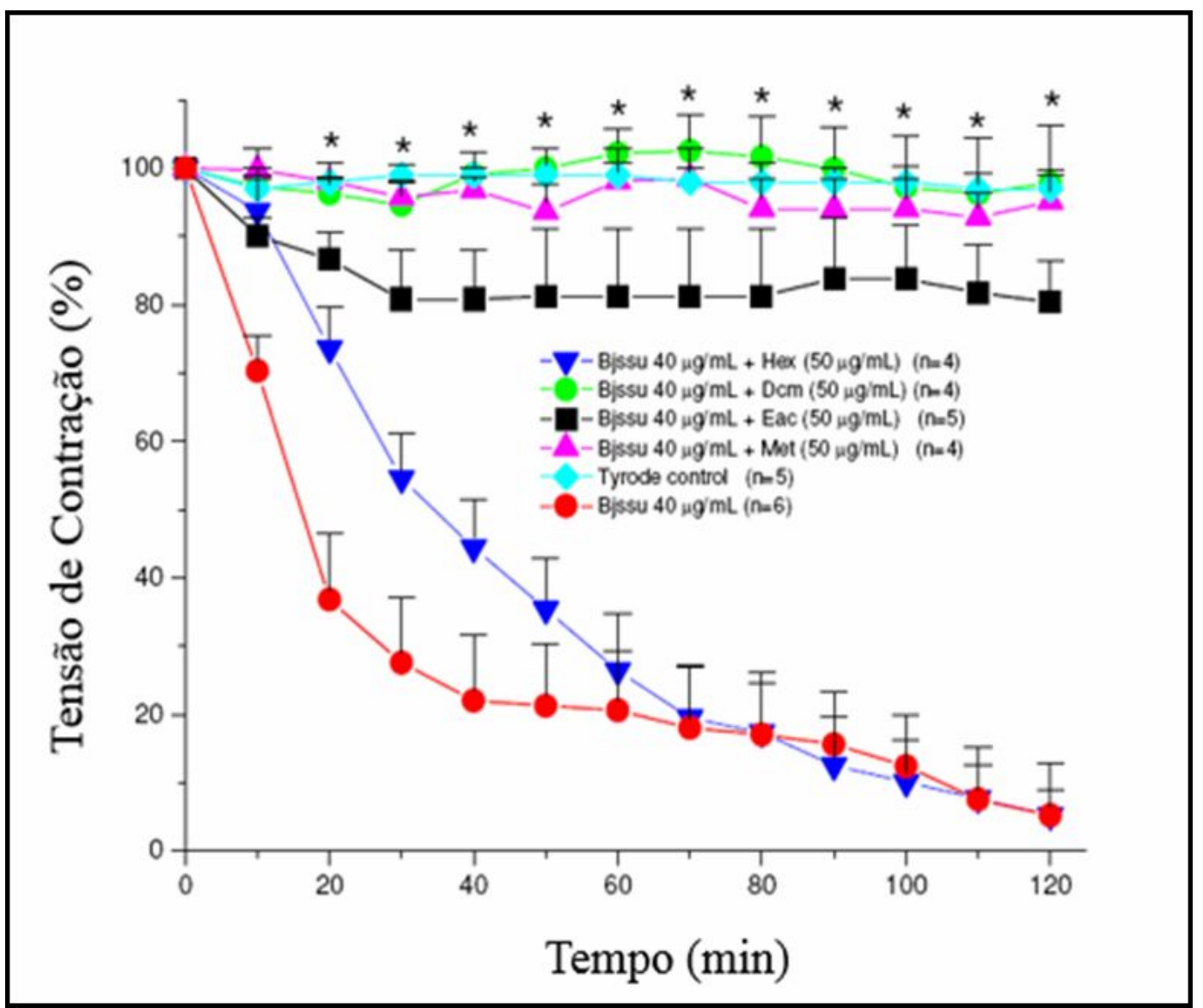

FIGURA 2 - Ensaios farmacológicos em preparações de diafragma de nervo frênico de rato com o objetivo de determinar a resposta de contração sob estímulos elétricos indiretos depois da incubação com veneno de $40 \mu \mathrm{g} / \mathrm{mL}$ de $B$. jararacussu (Bjssu) e $50 \mu \mathrm{g} / \mathrm{mL}$ de subextratos da casca do caule de Dipteryx alata Vogel. Substratos utilizados: hexano (Hex), diclorometano (Dcm), acetato de etila (Eac), metanol (Met). Observa-se a ação de $100 \%$ do bloqueio neuromuscular do veneno de $B$. jararacussu e diferença de atuação, após 20 minutos, nos subextratos $\mathrm{Dcm}$, Eac e Met.

Fonte: Adaptado Puebla et al., (2010). 


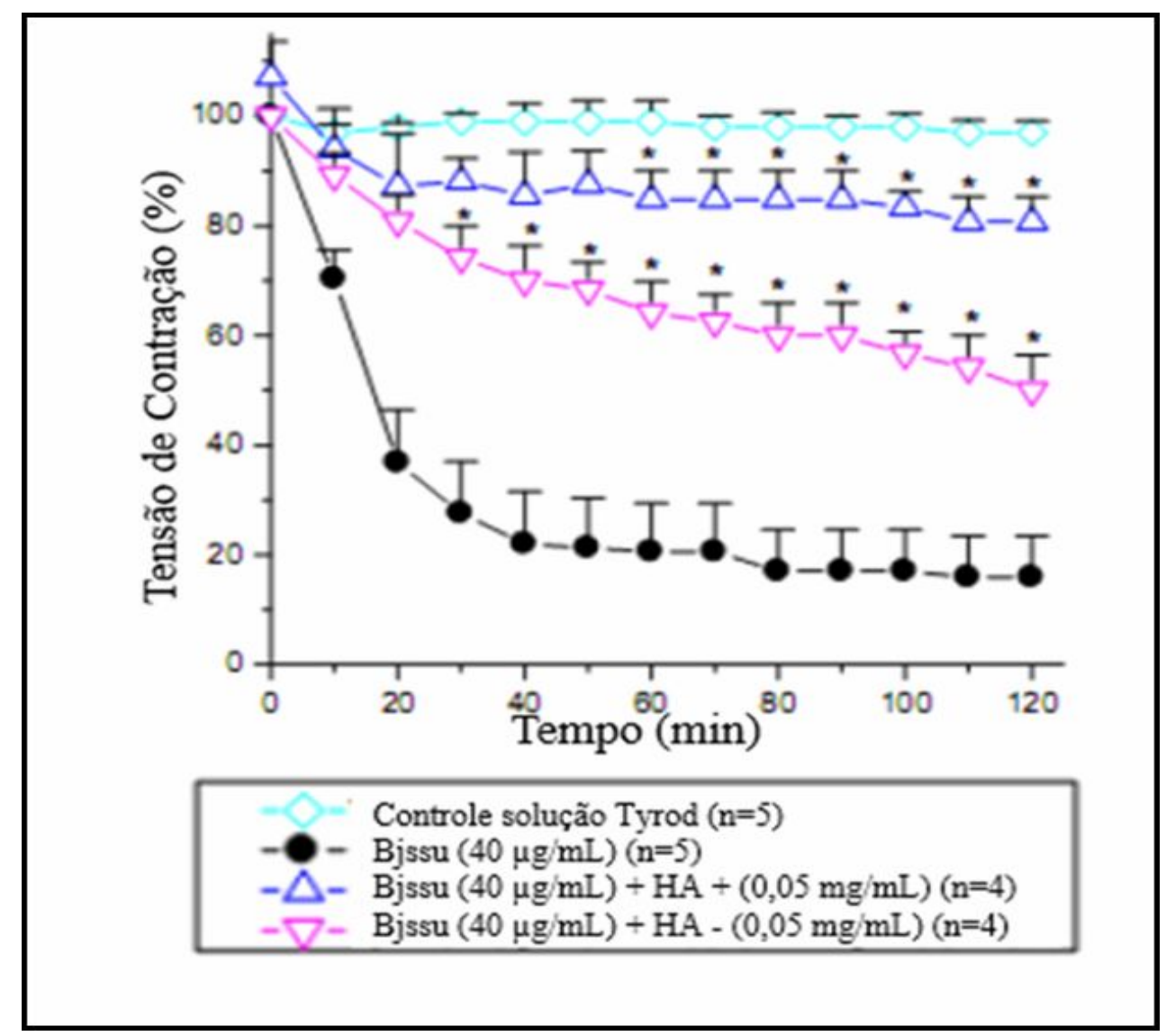

FIGURA 3 - Ensaio farmacológico para determinação do efeito de extrato hidroalcoólico de extratos da casca de Dipteryx alata com presença de tanino $(\mathrm{HA}+)(0,05 \mathrm{mg} / \mathrm{mL})$ e sem tanino (HA-) $(0,05 \mathrm{mg} / \mathrm{mL})$ incubados com Bjssu $(40 \mu \mathrm{g} / \mathrm{mL})$ e utilizados em preparações de nervo frênico-diafragma de ratos estimulados indiretamente. $\mathrm{O}$ extrato $(\mathrm{HA}+)$ melhorou a tensão de contração muscular, diminuindo o bloqueio neuromuscular causado pelo veneno de Bothrops jararacussu (Bjssu).

Fonte: Adaptado Nazato et al., (2010).

No mesmo estudo, avaliou-se veneno de Bjssu e $\mathrm{Cd}$ incubados com extratos de Hex, Dcm, Eac, Met em contração de tensão do nervo frênico-diafragma de ratos. Os resultados contra o veneno de Bjssu demonstram que o extrato Met apresentou melhores resultados e Dcm resultados parciais, sendo considerada a inibição mediada por taninos, ácidos fenólicos e flavonoides (Figura 4). Contra o veneno de $\mathrm{Cd}$ nenhum dos extratos demonstrou ação contra 0 bloqueio neuromuscular, comprovando a necessidade de identificar as substâncias presentes nos extratos e direcionar o tratamento para o bloqueio neuromuscular para cada espécie de cobras (NAZATO et al., 2010). 


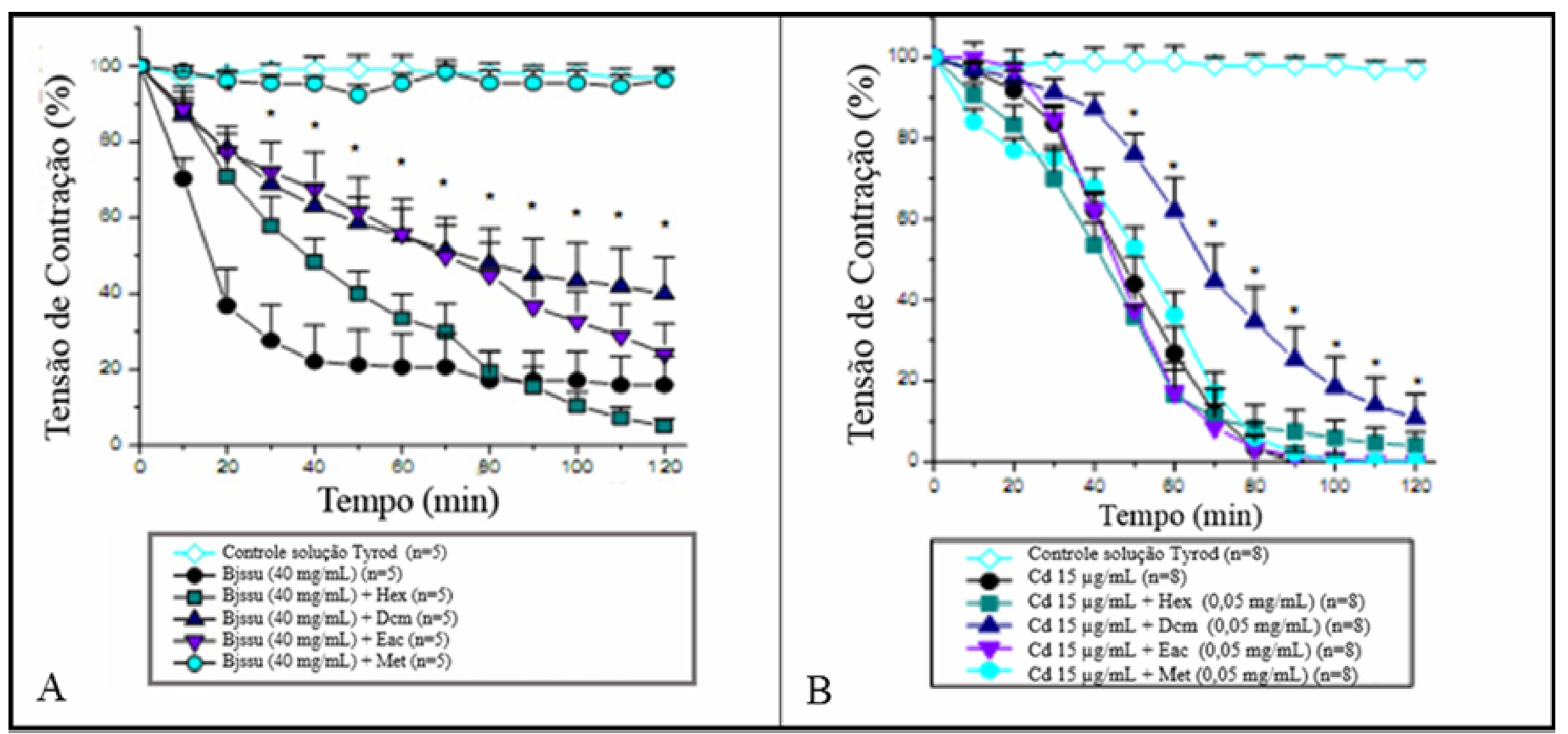

FIGURA 4 - Ensaios farmacológicos em preparações de nervo frênico-diafragma de rato para determinar a resposta de contração sob estímulos elétricos indiretos após incubação de $40 \mathrm{~g} / \mathrm{mL}$ de veneno de Bothrops jararacussu (Bjssu) (A) e Crotalus durissus terrificus (Cd) (B) préincubado durante 30 minutos com o extrato de casca de $D$. alata $(0,05 \mathrm{mg} / \mathrm{mL})$ utilizando solventes de hexano (Hex), diclorometano (Dcm), acetato de etilo (Eac), metanol (Met). Observa-se que o Met impediu 100\% do bloqueio neuromuscular causado pelo veneno de Bjssu e Dcm diminuiu parcialmente. Com Cd o Dcm atrasou o bloqueio, mas nenhum extrato causou ação de bloqueio neuromuscular.

Fonte: Adaptado Nazato et al., (2010).

Com o intuito de avaliar histologicamente os danos causados nos músculos do nervo frênico-diafragma após a exposição aos compostos tóxicos de venenos de cobra e o possível efeito protetor de extrato de $D$. alata, os autores escolheram o incubado Bjssu com extrato Met por ter apresentado melhores resultados. Três cortes histológicos do nervo foram preparados, um com a solução de Tyrod, outro exposto ao veneno de Bjssu e o terceiro de Bjssu inoculado com o extrato Met, com 120 minutos de exposição. Observando o tecido exposto a Tyrod as fibras danificadas não excediam $10 \%$ e as mudanças observadas eram diferentes dos danos causados por Bjssu, observou-se atrofia das fibras musculares, hipercontração, condensação e/ou lise de miofibrilas. No tecido com Bjssu e o extrato Met observou-se proteção dos danos celulares por não haver ruptura das membranas celulares (NAZATO et al., 2010). (Figura 5). 


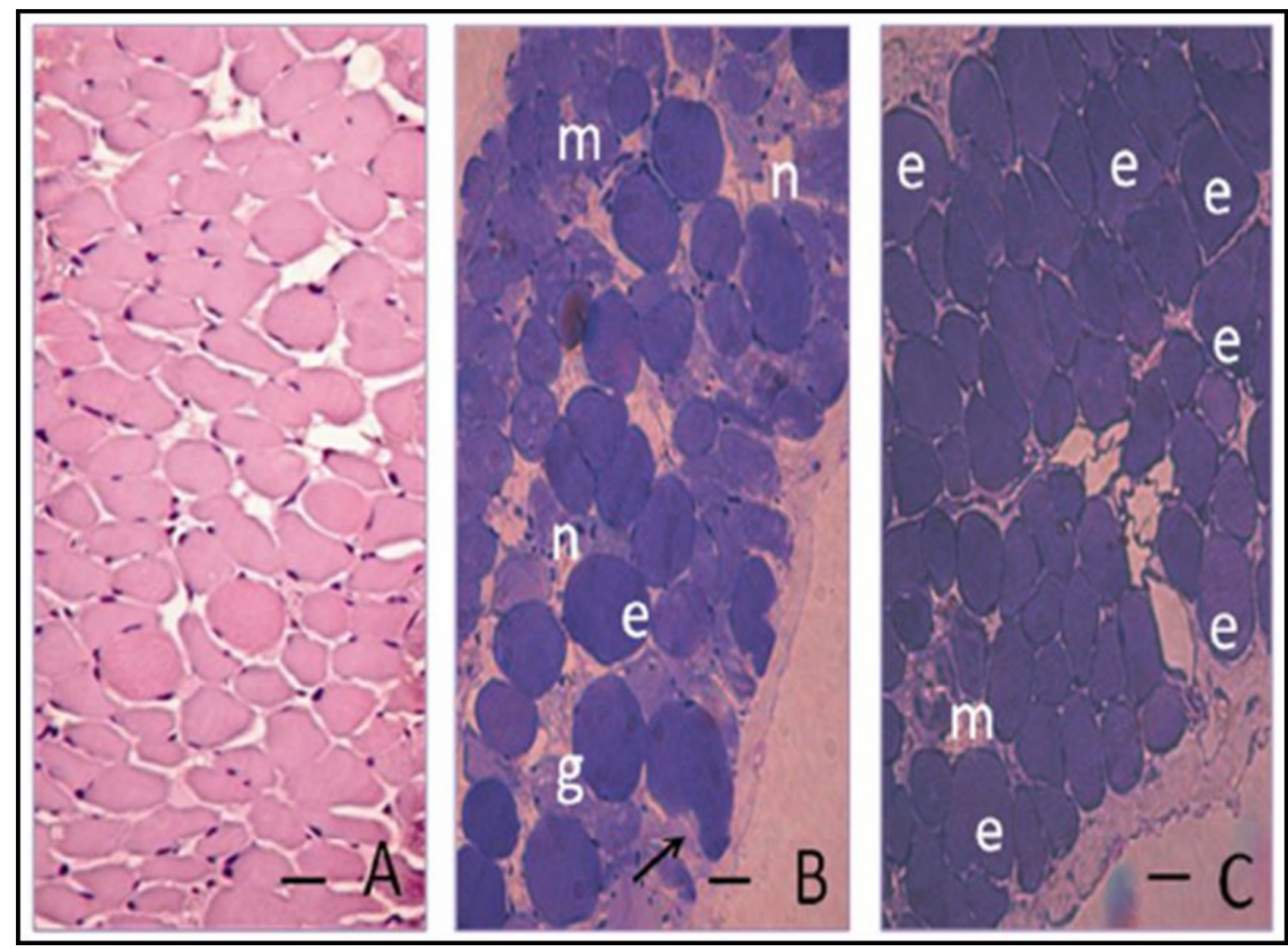

FIGURA 5 - Sessão de nervo frênico-diafragma após tratamento. A - Tratamento com solução de Tyrod. Perda de perfil poligonal de seção transversal de células típicas. B Tecido submetido a inoculação de veneno Bjssu. Presença de células necróticas $(\mathrm{m})$, fibras de tamanho heterogêneo com algumas apresentando aspecto edematoso (e) sem perfil transversal poligonal ou com lesões delta (flecha), células "fantasmas" ou com sarcolema purulento indicando ruptura de sarcolema (g), núcleos deslocados (n) indicando mionecrose grave. C - Tratamento com Bjssu e extrato Met. A maioria das células manteve seu perfil poligonal, a mionecrose ocorreu em menor quantidade $(\mathrm{m})$ e apenas alguns foram edematosos (e). O extrato impediu a ruptura de membrana apresentando proteção ao tecido.

Fonte: Adaptado Nazato et al., (2010).

Estudos científicos com plantas voltados para avaliações terapêuticas são considerados importantes não somente para a prospecção de novas moléculas terapeuticamente ativas, como também para auxiliar na conservação e investimentos nos biomas brasileiros (NAZATO et al., 2010).

\section{CONSIDERAÇÕES FINAIS}

A utilização de plantas medicinais como recurso terapêutico é uma prática legitimada e difundida e muitas plantas têm sido utilizadas cientificamente, como $D$ alata, ganhando espaço no campo da ciência e entre profissionais da saúde. Muitas destas plantas saíram da observação empírica e tem ganhado espaço em laboratórios de universidades com objetivo de garantir sua legitimidade. 
A amêndoa de $D$. alata foi utilizada em pesquisas com ratos e camundongos para avaliações de perfil lipídico e como antioxidante. Os resultados são promissores e comprovaram que o consumo diário da semente, mesmo em pequenas doses melhora algumas análises clínicas como glicemia e colesterol. Ã divulgação destes resultados podem contribuir para um aumento do consumo da semente, proporcionando aumento na produção e comercialização da amêndoa podendo proporcionar uma complemento na renda de produtores rurais e ainda uma melhora significativa na saúde humana. Pode haver uma contribuição na redução de doenças cardiovasculares. Estudos com as amêndoas em humanos também comprovaram redução em LDL-c devido a presença de ácidos graxos insaturados e compostos bioativos.

Existem também estudos com o óleo da amêndoa para produção de emulsão de gel para utilização como agente veicular de cosméticos de pele, demonstrando mais aplicabilidades da espécie, em área cosmética, além da alimentícia. A

folha de $D$. alata teve um primeiro estudo voltado para doenças para hiperpigmentação da pele que pode ser continuado em estudos mais complexos com melanócitos. Este seria uma importante utilização já que durante a maturação dos frutos as folhas caem e poderiam ser utilizadas como subproduto da espécie, sem prejudicar sua fisiologia.

Um dos únicos estudos publicados da polpa e da casca incluíram a utilização da semente como comparativo das três partes em inibições contra microrganismos. Dos três a casca e a amêndoa demonstraram melhores resultados e para a produção do fruto a utilização da casca do fruto, que geralmente é descartada pode gerar uma renda extra para a produção rural.

A casca do caule tem sido utilizada pela população na forma de chá ou embebida em vinhos para a utilização contra acidentes antiofídicos. A utilização popular foi comprovada com os bons resultados dos trabalhos de alguns pesquisadores que utilizaram a espécie em estudos com nervo frênico-diafragma de rato.

De todas as implicações terapêuticas pesquisadas com $D$. alata, nenhuma demonstrou a citotoxicidade de partes da planta em estudos cancerígenos e nem todas as partes da espécie forma utilizadas em pesquisas, como o endocarpo do fruto. Assim, entendemos como necessária e primordial estudos de outras partes do fruto de $D$. alata, bem como novos estudos referentes às implicações terapêuticas dessa importante espécie na área rural de Goiás.

\section{REFERÊNCIAS}

ALVIM, N. A. T.; FERREIRA, M.A.; CABRAL, I. E.; FILHO, A.J.A. O uso de plantas medicinais como recurso terapêutico: das influências da formação profissional às implicações éticas e legais de sua aplicabilidade como extensão da prática de cuidar realizada pela enfermeira. Revista Latino-am Enfermagem 2006 maio-junho; v. 14, n. 3, 2006. http://www.redalyc.org/html/2814/281421862003/

ARAÚJO, A. C. F.; ROCHA, J. C.; PARAISO, A. F.; FERREIRA, A. V. M.; SANTOS, S. H. S.; PINHO, L. Consumption of baru (Dipteryx alata) in the treatment of obese mice. Ciência Rural, Santa Maria. 2017; v.47; 02, e20151337. http://dx.doi.org/10.1590/0103-8478cr20151337. 
ARAÚJO, A. C.; GUIGUER, É. L.; BARBALHO, S. M.; BUENO, P. C.; LOPES, JA.; SILVA, B. F.; GIROTTO, L. C. PAULA, M. G.; ZEBER, P. V.; ALVARES, G. R. Phytochemical Characteristics of Seeds and Its Effects on the Intestinal Motility and Toxicity of Joannesia princeps. Journal of Medicinal Food, v. 19, n. 1, p. 68-72, 2016. doi: $10.1089 /$ jmf.2015.0071

BAILÃO, E. F. L. C.; DEVILLA, I. A.; CONCEIÇÃO, E. C.; BORGES, L. L. B. Bioactive compounds found in Brazilian Cerrado fruits. International Journal of Molecular Sciences, v. 16, n. 10, p. 23760-23783, 2015. doi:10.3390/ijms161023760.

BENTO, A. P. N.; COMINETTI, C.; FILHO, A. S.; NAVES, M. M. V. Baru almond improves lipid profile in mildly hypercholesterolemic subjects: a randomized, controlled, crossover study. Nutrition, Metabolism \& Cardiovascular Diseases. 2014; v.24, n. 12, p1330-1336. 10.1016/j.numecd.2014.07.002.

EKOR, M. The growing use of herbal medicines: issues relating to adverse reactions and challenges in monitoring safety. Frontiers in Pharmacology, v. 4, p. 177, 2014. doi: 10.3389/fphar.2013.00177

ESTEVES-PEDRO NM, BORIM T, NAZATO VS, SILVA MG, LOPES OS, SANTOS MG, DAL BELO CA, CARDOSO CRP, VARANDA EA, GROPPO FC, GERENUTTI, OSHIMA-FRANCO Y. In vitro and in vivo safety evaluation of Dipteryx alata Vogel Extract. Complementary and Alternative Medicina. 2012; 129.

FANK-DE-CARVALHO, S. M.; SOMAVILLA, N. S.; MARCHIORETTO, M. S.; BÁO, S. N. Plant structure in the Brazilian neotropical savannah species. In Biodiversity in Ecosystems-Linking Structure and Function; Lo, Y., Blanco, J.A., Roy, S., Eds.; InTech: Rijeka, Croatia, 2015; pp. 425-459. https://www.intechopen.com/books/biodiversity-in-ecosystems-linking-structure-andfunction/plant-structure-in-the-brazilian-neotropical-savannah-species

FERNANDES, D. C.; ALVES, A. M.; CASTRO, G. S. F.; JÚNIOR, A. A. J.; NAVES, M. M. V. Effects of baru almond and Brazil nut against hyperlipidemia and oxidative stress in vivo. Journal of Food Research, v. 4, n. 4, p. 38, 2015. http://dx.doi.org/10.5539/jr.v4n4p38.

FIORINI, A. M. R.; GUIGUER, E. L.; OSHIIWA, M.; MENDES C. G.; VIEITES, R. L.; CHIES, A. B.; OLIVEIRA, P. B.; SOUZA, M. S. S. S.; NICOLAU, C. C. T. Dipteryx alata Vogel May Improve Lipid Profile and Atherogenic Indices in Wistar Rats Dipteryx alata and Atherogenic Indices. Journal of Medicinal Food, v. 20, n. 11, p. 1121-1126, 2017. doi: 10.1089/jmf.2017.0052

MACIEL, M. A. M.; ANGELO, C. P.; VEIGA, V. F. J. Plantas medicinais: a necessidade de estudos multidisciplinares. Química Nova, v. 25, n. 3, p. 429-438, 2002. http://dx.doi.org/10.1590/S0100-40422002000300016.

MORAES, C.; ANJOS, J. L. V.; MARUNO, M.; ALONSO, A. ROCHA-FILHO, P. Development of lamellar gel phase emulsion containing baru oil (Dipteryx alata Vog.) 
as a prospective delivery system for cutaneous application. Asian Journal of Pharmaceutical Sciences. Volume 13, Issue 2, March 2018, Pages 183-190. https://doi.org/10.1016/j.ajps.2017.09.003

NAZATO, V. S.; RUBEM-MAURO, L.; VIEIRA, N. A. G.; ROCHA-JUNIOR, D. S.; SILVA, M. G.; LOPES, P. S.; DAL-BELO CHÁRISTON, A. D.; COGO, J. C.; SANTOS, M. G.; CRUZ-HÕFLING, M. A.; OSHIMA, Y. In Vitro Antiophidian Properties of Dipteryx alata Vogel Bark Extracts. Molecules. 2010; 15, 5956-5970. doi:10.3390/molecules15095956.

PUEBLA, P.; OSHIMA-FRANCO, Y.; FRANCO, L. M.; DOS SANTOS, M. G.; SILVA, R. V.; RUBEM-MAURO, FELICIANO A. S. Chemical Constituents of the Bark of Dipteryx alata Vogel, an Active Species against Bothrops jararacussu Venom. Molecules. 2010; 15, 8193-8204; doi:10.3390/molecules15118193.

SANTOS, F. B.; RAMOS, M. I. L.; MIYAGUSKU L. Antimicrobial activity of hydroalcoholic extracts from genipap, baru and taruma. Ciência Rural, Santa Maria. 2017; v.47: 08, e20160252. http://dx.doi.org/10.1590/0103-8478cr20160252.

SILVERIO, M. D. O.; CASTRO, C. F. S.; MIRANDA, A. R. Avaliação da atividade antioxidante e inibitória da tirosinase das folhas de Dipteryx alata Vogel (Baru). Revista Brasileira de Plantas Medicinais, Botucatu. 2013; v.15, n.1, p.59-65. http://dx.doi.org/10.1590/S1516-05722013000100008.

SILVA, V. A.; FREITAS, A. F. R.; PEREIRA, M. S. V.; OLIVEIRA, C. R. M.; DINIZ, M. F. F. M.; PESSÔA, H. L. F. Eficácia antifúngica dos extratos da Lippia sidoides Cham. e Matricaria recutita Linn. Sobre leveduras do Gênero Candida. BioFar 2011, 5, 18-23. http://sites.uepb.edu.br/biofar/download/v5n12011/atividade_antifungica_dos_extratos.pdf

SIQUEIRA, E. M. A.; MARIN, A. M. F.; CUNHA, M. S. B.; FUSTINONI, A. M.; SANT'ANA, L. P.; ARRUDA, S. F. Consumption of baru seeds [Dipteryx alata Vog.], a Brazilian savanna nut, prevents iron-induced oxidative stress in rats. Food Research International. 2012; 45, 427-433. 10.1016/j.foodres.2011.11.005

SOUZA, C. D.; FELFILI, J. M. Uso de plantas medicinais na região de Alto Paraíso de Goiás, GO, Brasil. Acta Botanica Brasilica, v. 20, n. 1, p. 135-142, 2006. doi: 10.1590/S0102-33062006000100013

SOUZA, R. G. M.; GOMES, A. C.; CASTRO, I. A.; MOTA, J. F. A baru almondenriched diet reduces abdominal adiposity and improves high-density lipoprotein concentrations: A randomized, placebo-controlled trial. Nutrition (Burbank, Los Angeles County, Calif.), v. 55, p. 154-160, 2018. doi: 10.1016/j.nut.2018.06.001

WONG, S. K.; CHIN, K. Y.; SUHAIMI, F. H.; Animal models of metabolic syndrome: a review. Nutrition \& Metabolism, v. 13, n. 1, p. 65, 2016. doi: 10.1186 / s12986-0160123-9 
WU, A.; CORESH, J.; SELVIN, E.; TANAKA, H.; HEISS, G.; HIRSCH, A. T.; MATSUSHITA, K. Lower extremity peripheral artery disease and quality of life among older individuals in the community. Journal of the American Heart Association, v. 6, n. 1, p. e004519, 2017. doi: 10.1161/JAHA.116.004519. 